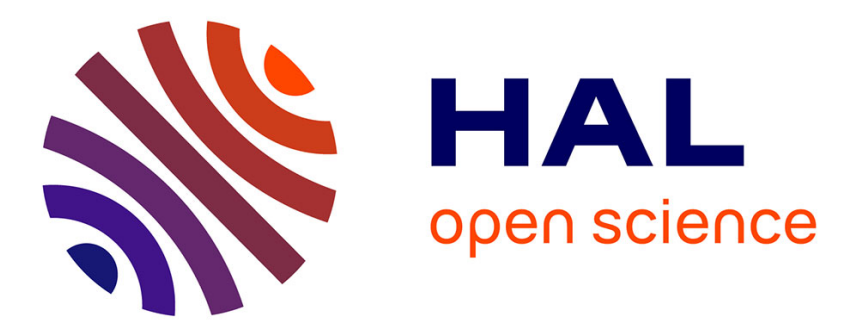

\title{
Dynamics of second-harmonic generation in a photovoltaic photorefractive quadratic medium
}

Frederico Pettazzi, V. Coda, Gil Fanjoux, Mathieu Chauvet, Eugenio Fazio

\section{To cite this version:}

Frederico Pettazzi, V. Coda, Gil Fanjoux, Mathieu Chauvet, Eugenio Fazio. Dynamics of secondharmonic generation in a photovoltaic photorefractive quadratic medium. Journal of the Optical Society of America B, 2010, 27 (1), pp.1-9. 10.1364/JOSAB.27.000001 . hal-00453839

\section{HAL Id: hal-00453839 \\ https://hal.science/hal-00453839}

Submitted on 22 Apr 2021

HAL is a multi-disciplinary open access archive for the deposit and dissemination of scientific research documents, whether they are published or not. The documents may come from teaching and research institutions in France or abroad, or from public or private research centers.
L'archive ouverte pluridisciplinaire HAL, est destinée au dépôt et à la diffusion de documents scientifiques de niveau recherche, publiés ou non, émanant des établissements d'enseignement et de recherche français ou étrangers, des laboratoires publics ou privés.

\section{(c)(1)}

Distributed under a Creative Commons Attribution| 4.0 International License 


\title{
Dynamics of second-harmonic generation in a photovoltaic photorefractive quadratic medium
}

\author{
Federico Pettazzi, ${ }^{1,4, *}$ Virginie Coda, ${ }^{2}$ Gil Fanjoux, ${ }^{3}$ Mathieu Chauvet, ${ }^{3}$ and Eugenio Fazio ${ }^{1}$ \\ ${ }^{1}$ Dipartimento di Energetica, Universitá di Roma La Sapienza and CNISM, via A. Scarpa 16, 00161 Rome, Italy \\ ${ }^{2}$ Laboratoire Matériaux Optiques, Photonique et Systémes, UMR CNRS 7132, Université Paul Verlaine, \\ Metz-Supélec, 2 rue Edouard Belin, 57070 Metz, France \\ ${ }^{3}$ Département d'Optique, Institut FEMTO-ST, UMR CNRS 6174, Université de Franche-Comté, \\ 25030 Besançon, France \\ ${ }^{4}$ Current address: European Space Agency, ESTEC-TEC-MME, Noordwijk, The Netherlands \\ *Corresponding author: federico.pettazzi@gmail.com
}

\begin{abstract}
We investigate second-harmonic generation (SHG) in a photorefractive photovoltaic medium such as lithium niobate. Our numerical model reveals the complex dynamics of the parametric process during the buildup of the index modification due to the photorefractive (PR) nonlinearity. We investigate a condition in which no external field is applied to the crystal, resulting in a defocusing nonlinearity, as well as the case in which an external bias is applied, producing a self-focusing effect that can enhance the conversion efficiency of the parametric process. We also find the conditions for the initial phase matching and for the background illumination leading to a stable self-confined propagation of the second-harmonic generated light. The developed numerical model shows that as a general case SHG in a self-focusing PR medium results in mode beating inside the generated waveguide, as experimentally observed.
\end{abstract}

\section{INTRODUCTION}

Photorefractivity is widely used to induce quasipermanent index structures in materials by means of light [1]. In the last 20 years many interesting phenomena based on photorefractive (PR) effect were predicted and analyzed experimentally, such as the propagation of monomode [2] and multimode [3] spatial solitons and, more recently, the study of light propagation in nonlinear quasi-periodic media $[4,5]$. PR solitons are also useful tools to write passive structures, such as matrices $[6,7]$ and junctions [8], inside the material, since they are stable against perturbations and can be permanently fixed under certain conditions in the crystal [9]. Moreover they can be induced using low intensity visible light and are wavelength sensitive. Consequently, solitons induced by visible light can be used as a waveguide for high intensity infrared beams that typically do not experience any PR effect [10].

Many PR crystals possess high second-order susceptibility, and thus can be used for parametric processes such as second-harmonic generation (SHG) [11], difference frequency generation [12], and parametric oscillation [13]. Quadratic nonlinearity can also display spatial selffocusing via cascading effects [14-16].

However, usually photorefraction is considered a detrimental effect for parametric processes such as SHG, particularly in lithium niobate (LN) crystals, which display a defocusing nonlinearity due to the presence of the photovoltaic (PV) effect, causing beam distortion and consequently unstable nonlinear conversion [17]. Indeed, the PR effect is typically observed even in nominally undoped
$\mathrm{LN}$ samples due to the presence of intrinsic and extrinsic defects in the crystal lattice. Low defect concentrations are sufficient to observe PR nonlinear index modifications and beam distortion with microwatt visible beams. Therefore, even in a low conversion efficiency parametric process, the PV effect can seriously affect the shape of the interacting beams and the overall harmonic generation.

While the PR effect is adverse for the SHG in the undoped LN crystal it is possible to take advantage of it to improve and control parametric processes. Such a goal can be reached if the PR effect leads to a stable selfconfinement of traveling beams. We would thus benefit from undistorted beams, higher parametric efficiency due to beam confinement, and a self-alignment process. Converting original defocusing to focusing effect is possible by the application of an external electrical bias to the LN crystal [18], which can end up in the formation of a selfinduced waveguide in the material. Parametric interactions inside self-induced waveguides can therefore be an interesting technique to improve conversion efficiency while avoiding the defocusing effect occurring in the LN crystal, as experimentally demonstrated for Fe:LN in a phase mismatched interaction inside a 1D PV soliton induced waveguide [19]. However, interplay between nonlinear effects necessitates the development of a time dependent model in order to explain the observed dynamics and above all to be used as a tool to determine conditions giving an efficient steady-state regime.

To tackle this problem, we present in this paper a $(1+1)$ D time dependent numerical analysis of the SHG in a photorefractive photovoltaic (PR-PV) material. We con- 
sider a single carrier PR-PV model valid for $\mathrm{LN}$, for which charge transport can be due to both the PV effect and an externally applied bias field. Such a configuration supports both bright and dark solitons of the screening PR-PV family [20]. As is well known, when a visible laser beam travels inside such a sample, photogeneration and displacement of electrons from filled traps cause the formation of a space charge field in the material [21]. This space charge field is responsible for a modulation of the refractive index via the linear electro-optic effect. In $\mathrm{LN}$, lattice asymmetry induces a PV electric field, which causes a local decrease in the refractive index in the illuminated region. When the crystal is externally biased in the direction of the $c$ axis a drift current can counteract and overpass the PV current, thus resulting in a local increase in the refractive index in the shined part of the medium, a necessary condition for the formation of bright solitons $[18,20]$.

LN also possesses high second-order optical nonlinearity and consequently can generate visible light from near infrared sources via quadratic processes [11]. While infrared light cannot induce $\mathrm{PR}$ effect due to the low photon energy, the second-harmonic ( $\mathrm{SH})$ beam is able to generate charges from deep centers, thus giving rise to a nonlinear index modification, which can be sensed also by the infrared fundamental harmonic (FH) beam. The complex interplay between quadratic and $\mathrm{PR}$ nonlinear responses can eventually lead to a stable self-confined propagation of the two beams inside the LN crystal. A similar study was previously initiated in $\mathrm{PR} \mathrm{KNbO}_{3}$ both from the experimental [22] and theoretical [23] points of view but it only concerned steady-state soliton conditions. On the contrary, our model takes into account the time dependence of the PR buildup and considers the presence of the PV nonlinear defocusing. It thus provides a way to better comprehend the complex dynamics of the process and to determine the parameters to possibly avoid beam distortion and defocusing reaching a stable self-confinement of both interacting beams.

\section{THEORY AND NUMERICAL APPROACH}

\section{A. Phase Matching in Lithium Niobate Using Temperature and Applied Field}

We consider a noncritical type-I configuration for the SHG involving a $\mathrm{FH}$ beam at $1064 \mathrm{~nm}$ ordinary polarized and an extraordinary polarized $\mathrm{SH}$ beam at a wavelength of $532 \mathrm{~nm}$. For these wavelengths LN is phase matchable in noncritical configuration using temperature [24] and electric field [25] tuning, without spatial walk-off. The generalized Sellmeier equations derived by Schlarb and Betzler in [26] permit one to calculate the phase matching (PM) conditions as functions of temperature, wavelength, and lithium content. In the following, we will consider a PM temperature of $-4^{\circ} \mathrm{C}$ for our sample at $1064 \mathrm{~nm}$, in order to match results obtained in the experimental part. However, when an external bias is applied to the sample in the direction of the $c$ axis, the extraordinary (e) and the ordinary (o) indices vary due to the electro-optic coefficients, respectively, $r_{33}$ for the e-polarized $\mathrm{SH}$ beam and $r_{13}$ for the o-polarized FH beam. This electrical bias then causes a shift of the PM temperature.
In Fig. 1 we present the variation in the PM temperature as a function of the external field by means of the electro-optic effect. This plot has been obtained by combining the Sellmeier equations with the usual set of equations for the electro-optics effect, yielding the variation in the phase mismatch $\left(\Delta n=n_{e}^{2 \omega}-n_{o}^{\omega}\right)$ as a function of both temperature and static field. Subsequently, the equation $\Delta n(T, E)=0$ has been solved to derive the curve displayed in Fig. 1, showing that the PM temperature increases linearly with the external bias applied along the $c$ axis. In Fig. 1 the PM curve separates the plot in an upper region, where a positive mismatch holds $(\Delta n>0)$, and a lower region, where $\Delta n<0$. In the inset of Fig. 1 the computed PM curve is reported for a $5 \mathrm{~mm}$ crystal in the noncritical SHG configuration with no applied field.

\section{B. Numerical Model}

In LN no PR effect occurs at $1064 \mathrm{~nm}$, since the photon energy is not sufficient to ionize deep donors that are present in the forbidden gap of the material. However, green $\mathrm{SH}$ light is absorbed by the deep traps and induces a PR effect in the material.

In the $(1+1) \mathrm{D}$ model we consider a geometry in which the beams propagate in the $z$ direction and linearly diffract in the $x(c)$ direction. The crystal optical $c$ axis and the direction of the applied field are parallel to the $x$ direction.

In accordance with the above geometry, the coupled wave equations that express the $\mathrm{FH}$ and $\mathrm{SH}$ amplitude profile evolutions $E_{1}$ and $E_{2}$ in the presence of the PR effect using the slowly varying envelope approximation are [23]

$$
\begin{gathered}
2 j k_{1} \frac{\partial E_{1}}{\partial z}=\frac{\partial^{2} E_{1}}{\partial x^{2}}+2\left(\frac{\omega}{c}\right)^{2} d E_{1}^{*} E_{2} e^{-j \Delta k z}-\frac{\omega^{2}}{c^{2}} r_{13} n_{o}^{4} E_{s c}\left(I_{2}\right) E_{1}, \\
2 j k_{2} \frac{\partial E_{2}}{\partial z}=\frac{\partial^{2} E_{2}}{\partial x^{2}}+\left(\frac{2 \omega}{c}\right)^{2} d E_{1}^{2} e^{j \Delta k z}-\frac{2 \omega}{c^{2}}{ }^{2} r_{33} n_{e}^{4} E_{s c}\left(I_{2}\right) E_{2},
\end{gathered}
$$

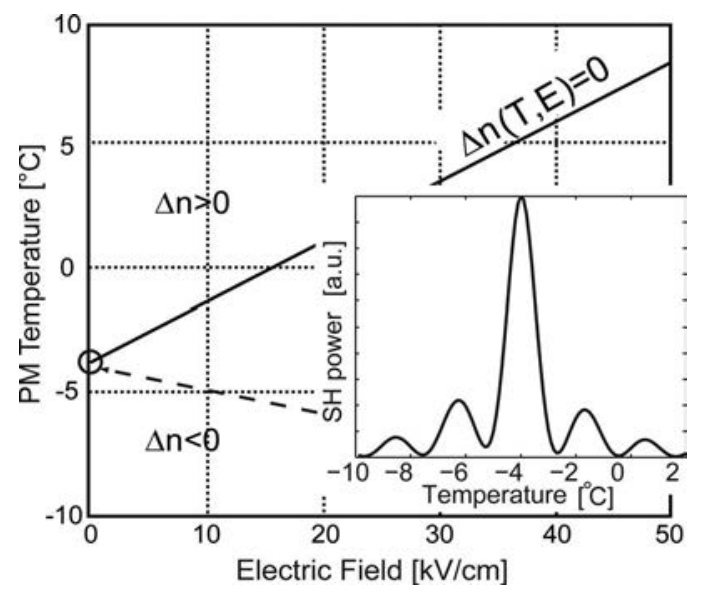

Fig. 1. Variation in the PM condition $\left(\Delta n=n_{e}^{2 \omega}-n_{o}^{\omega}\right)$ as a function of applied external field assuming a lithium content $c_{L i}=48.41 \%$. In the inset the width of the resonance versus the temperature is reported for the case with no applied field. 
where the $\mathrm{FH}$ and $\mathrm{SH}$ optical fields are, respectively, given by $E_{\mathrm{FH}}=E_{1} \exp j\left(\omega t-k_{1} z\right)$ and $E_{\mathrm{SH}}=E_{2} \exp j(2 \omega t$ $-k_{2} z$ ). In Eqs. (1) $k_{1}$ and $k_{2}$ are the wave vectors of the $\mathrm{FH}$ and $\mathrm{SH}$ beams; $d$ is the quadratic nonlinear coefficient that takes into account crystal orientation and beam polarization; $r_{13}$ and $r_{33}, n_{o}$ and $n_{e}$ are the electro-optic coefficients and refractive indices seen by $\mathrm{FH}$ and $\mathrm{SH}$ beams, respectively; and $\Delta k=k_{2}-2 k_{1}$ is the wave vector mismatch between interacting waves. Finally, $E_{s c}\left(I_{2}\right)$ is the space charge field distribution induced by the $\mathrm{SH}$ optical field via the PR-PV effect, which is linked to the optical intensity of the SH light $I_{2}$. Equations (1) are solved numerically to derive the amplitude distribution of the optical field for both the interacting waves, provided the space charge field can be calculated. The generated $\mathrm{SH}$ light modifies the refractive index of the material as described by the usual set of PR Kuktharev equations [20]. According to the experimental conditions, we can assume that the density of ionized deep donors $N_{D}^{+}$is much larger than the density of free electrons $N_{e}$ and generation processes are faster in comparison to the charge movements [27]. At last we neglect the diffusion term, which leads to the single differential equation giving the temporal evolution of the space charge field [28],

$$
\epsilon_{0} \epsilon \frac{\partial E_{s c}}{\partial t}=-e \mu A\left(I_{2}+I_{d}\right) E_{s c}+\beta_{p h}\left(N_{D}-N_{A}\right) I_{2}+e \mu A I_{d} E_{\text {ext }}
$$

In Eq. (2) $\epsilon$ is the relative dielectric constant of the medium; $e$ is the electric charge; $\mu$ is the electron mobility; $I_{d}$ is the dark irradiance, $N_{D}$ and $N_{A}$ are, respectively, the deep donor and the shallow acceptor densities; $E_{\text {ext }}$ is the external applied field; $\beta_{p h}$ is the PV coefficient; and $A$ $=s\left(N_{D}-N_{A}\right) /\left(\gamma N_{A}\right)$, where $s$ is the photoionization cross section and $\gamma$ is the recombination constant. A list of the parameters used for the simulation on the LN crystal is reported in Table 1.

To start, the $\mathrm{SH}$ and $\mathrm{FH}$ beam distributions are calculated in an unperturbed medium with a beam propagation method algorithm, with a spatial sampling step at least 20 times smaller than the $\mathrm{FH}$ beam diameter at the input. Considering an illumination time $\delta t$ much shorter than the PR response time, the space charge field variation is then derived from Eq. (2). These two steps are then repeated iteratively until steady state is reached. In the following simulations the temporal coordinate used $(\tau)$

Table 1. LN Parameter Values Used in the Simulations

\begin{tabular}{lcc}
\hline Parameter & Value & Reference \\
\hline$\epsilon$ & 32 & {$[28]$} \\
$\mu$ & $0.74 \mathrm{~cm}^{2} / \mathrm{s}$ & {$[28]$} \\
$N_{A}$ & $5 \times 10^{17} \mathrm{~cm}^{-3}$ & {$[28]$} \\
$N_{D}$ & $1.1 N_{A}$ & {$[28]$} \\
$\gamma$ & $1.64 \times 10^{-8} \mathrm{~cm}^{3} / \mathrm{s}$ & {$[28]$} \\
$I_{d}$ & $1 \times 10^{-9} \mathrm{~W} / \mathrm{cm}^{2}$ & {$[28]$} \\
$d$ & $-4.5 \mathrm{pm} / \mathrm{V}$ & {$[29]$} \\
$r_{33}$ & $30 \mathrm{pm} / \mathrm{V}$ & {$[17]$} \\
$r_{13}$ & $6.8 \mathrm{pm} / \mathrm{V}$ & {$[17]$} \\
\hline
\end{tabular}

is normalized to the dielectric relaxation time $T_{D}$ $=\left(\epsilon_{0} \epsilon \gamma N_{A}\right) /\left(e \mu s I_{d}\left(N_{D}-N_{A}\right)\right)$ that represents the time scale characteristic of the PR-PV decay in the dark.

The generated green light is quantified integrating the output $\mathrm{SH}$ intensity in the transversal coordinate at any temporal step. The obtained value is then normalized to the output $\mathrm{SH}$ power when the perfect $\mathrm{PM}$ is obtained and no $\mathrm{PR}$ effect is present, in order to provide a means to quantify the effect of the nonlinear beam modification on the parametric conversion efficiency. Such a normalized $\mathrm{SH}$ power is independent of the chosen sample size. Note that the cancellation of the PR response in $\mathrm{LN}$ is possible either by increasing the temperature of the sample or by using a background illumination much stronger than the $\mathrm{SH}$ generated light.

\section{RESULTS AND DISCUSSION}

In a PR-PV crystal, the propagation of a visible spatially nonuniform light beam causes a local modification in the refractive index across the light path due to the buildup of the space charge field. According to the sign of the nonlinear response, the refractive index change induces a modification of the beam intensity profile. However, when the visible beam propagating inside the crystal arises from a nonlinear quadratic effect like the SHG, the dynamics of the whole process is far more complicated than in normal cases.

In fact, the $\mathrm{SH}$ signal generated is partially absorbed by the deep traps, inducing a space charge field and ultimately a local refractive index modification sensed by both $\mathrm{FH}$ and $\mathrm{SH}$ lights. At the same time, the modification of the index also affects the PM conditions via a local change in the phase velocities of both beams, with a process known as the nonlinear self-phase-matching (NLSPM) [17].

As a result, the conversion efficiency of the quadratic process is expected to vary due to two different mechanisms driven by the PR-PV effect: the spatial beam modification, which affects the intensity profile of the $\mathrm{FH}$ and $\mathrm{SH}$ beams, and the change in PM conditions, which instead modifies the phase relationship between the interacting waves. Optimizing the SHG process in a PR-PV crystal would therefore imply finding a condition at steady state in which a perfect PM is achieved and at the same time the beam is spatially confined in order to increase the FH beam intensity.

\section{A. NLSPM and Defocusing Nonlinearity}

In typical experiments using the LN crystal, the condition leading to perfect PM and confined output beam cannot be reached, because the $\mathrm{PV}$ effect induces a nonlinear defocusing that broadens the $\mathrm{SH}$ and FH outputs [17,30], compromising the beam quality and the conversion efficiency. To describe this common situation, we first study the case with no external bias applied to the LN crystal and we consider a $\mathrm{FH}$ beam focused to a $15 \mu \mathrm{m}$ diameter at the input face of a $5 \mathrm{~nm}$ long sample, generating $\mathrm{SH}$ light along propagation. In this configuration, the PV effect will be induced by the $\mathrm{SH}$ beam. In Fig. 2 we illustrate the PM diagram for the present case, assuming a crystal temperature giving an initial positive mismatch. 


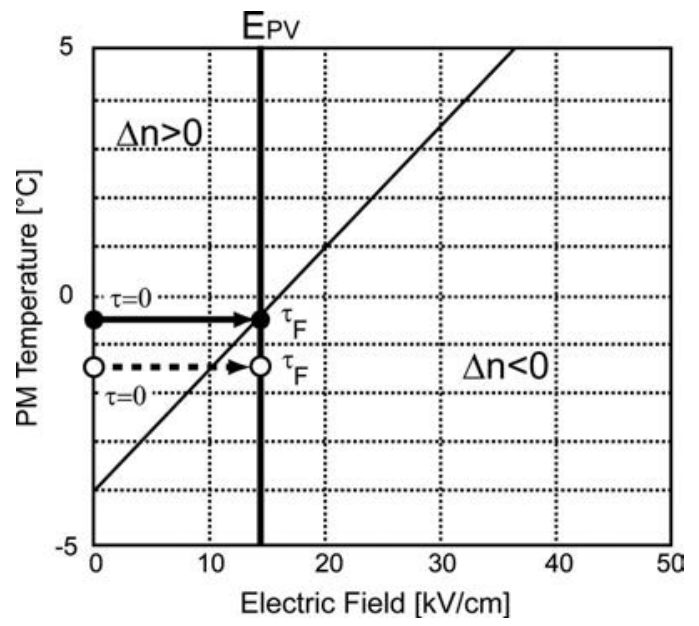

Fig. 2. Variation in the PM condition due to the NLSPM process given by the PV effect. The buildup of the space charge field can cause the complete recovery of the PM condition (continuous line arrow) or induce a switch from a positive to a negative mismatch (dashed line arrow), depending on the initial temperature (mismatch).

At the beginning $(\tau=0)$ the PM is only determined by the temperature of the crystal since $E_{\text {ext }}=0$. However, as the space charge field starts to rise due to the PV effect, the PM point moves closer to perfect matching and compensates the initial mismatch, until the steady-state regime is reached $\left(\tau=\tau_{F}\right)$, for which the space charge field has reached the maximum value of $E_{\mathrm{PV}}$. This value corresponds to the PV field at saturation, which is linked to the physical parameters of the material via the relation $E_{\mathrm{PV}}$ $=\left(\beta_{p h} \gamma N_{A}\right) /(q \mu s)$.

Thus, it follows that perfect PM at steady state $\left(\tau=\tau_{F}\right)$ can be reached when the initial positive phase mismatch is completely compensated by the rise of the space charge field induced by the PV effect as shown in Fig. 2 (continuous line arrow). To demonstrate this point, a simulation is carried out with an initial positive mismatch $\left(T=-0.5^{\circ} \mathrm{C}\right)$ between $\mathrm{FH}$ and $\mathrm{SH}$ beams, which can be exactly compensated by the PV field buildup. The FH intensity is $I_{1}(z=0)=1 \times 10^{4} \mathrm{~W} / \mathrm{cm}^{2}$ at the entrance face and the PV field at saturation is set to $E_{\mathrm{PV}}=14 \mathrm{kV} / \mathrm{cm}$, consistent with what are being used to model the experimental part.

Oscillating behaviors along propagation, typical of mismatched interactions, are visible in the SH intensity distribution at $\tau=0$ [Fig. 3(a)], together with the linear diffraction. Subsequently, the buildup of the PV field causes a local decrease in the index, which induces a nonlinear defocusing of the SH beam [Figs. 3(b) and 3(c)]. Simultaneously we observe that the number of oscillations related to the initial mismatch decreases, testifying that the system is evolving closer to the PM condition, following the continuous line arrow in the PM diagram (Fig. 2).

The corresponding evolution of the normalized $\mathrm{SH}$ power as a function of time is reported in Fig. 4 (continuous curve): the increasing generated SH power is the result of the recovery of PM conditions through the NLSPM process. At steady state the perfect PM condition is reached, as predicted in Fig. 2, for a temperature of $T=-0.5^{\circ} \mathrm{C}$ and an electric field of $E_{s c}=E_{\mathrm{PV}}=14 \mathrm{kV} / \mathrm{cm}$. The maximum SH intensity obtained is $I_{\mathrm{SH}}^{\max }=3.4 \mathrm{~W} / \mathrm{cm}^{2}$, which is not optimized at all due to the presence of a diffracting beam.

The SH power evolution can be totally different if the initial PM condition is not completely compensated by the space charge field buildup. For instance, when a weaker initial positive mismatch is chosen $\left(T=-1.5^{\circ} \mathrm{C}\right.$ in Fig. 2 dashed line arrow), the NLSPM effect can cause a change from positive to negative mismatch, thus passing temporarily through the perfect matching condition, as indicated by the peak in the plot of the SH power as a function of time (Fig. 4, dashed curve). In this case, a slightly lower maximum $\mathrm{SH}$ intensity is obtained during the process compared with the previous conditions ( $I_{\mathrm{SH}}^{\max }$ $=2.3 \mathrm{~W} / \mathrm{cm}^{2}$ ). Results presented in Fig. 4 are in accordance with experimental investigations performed in [17], where the initial PM condition is varied through angular tuning.

\section{B. NLSPM and Focusing Nonlinearity}

The nonlinear defocusing due to the PV effect can be reversed when an external field is applied to the crystal so that the buildup of the PR space charge field will result in a local decrease in the total field in the illuminated area, due to the screening of the applied field [18]. The total field will then generate a local increase in the refractive index and a self-focusing effect for both $\mathrm{SH}$ and FH lights, bringing the possibility to increase the SHG efficiency by the confinement of beams over a long distance. In Fig. 5 the PM diagram in the presence of an applied field is shown. The initial PM conditions are now imposed by both the crystal temperature and the external applied field.

If initially $(\tau=0)$ the temperature and the applied field are set to give an initial moderate negative mismatch, the

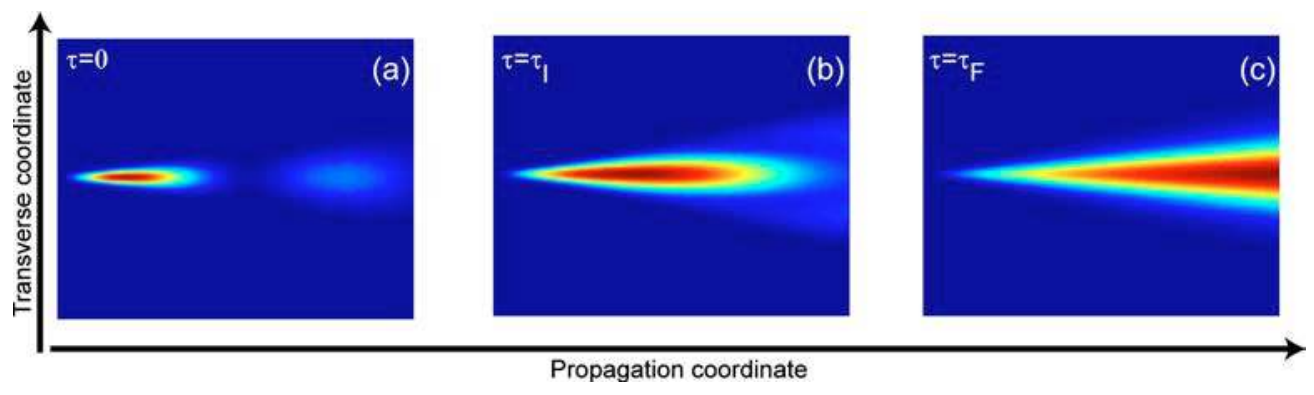

Fig. 3. (Color online) Temporal evolution of the SH light distribution in the sample for a defocusing nonlinearity at three characteristic times. Parameters of the simulation are $T=-0.5^{\circ} \mathrm{C}, E_{\mathrm{PV}}=14 \mathrm{kV} / \mathrm{cm}$, and $I_{1}(z=0)=1 \times 10^{4} \mathrm{~W} / \mathrm{cm}^{2}$. The time of each image is reported in Fig. 4 (continuous curve). 


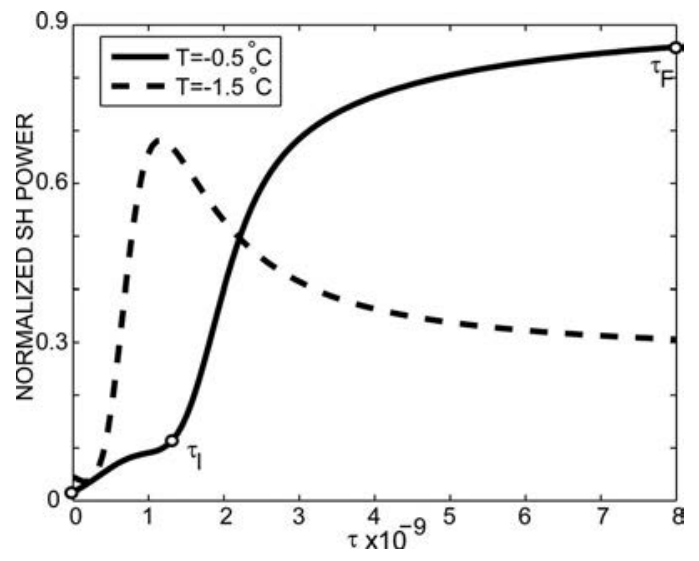

Fig. 4. SH output power evolution for two different temperatures: $T=-0.5^{\circ} \mathrm{C}$ (continuous curve) and $T=-1.5^{\circ} \mathrm{C}$ (dashed curve). Other parameters are $E_{\mathrm{PV}}=14 \mathrm{kV} / \mathrm{cm}, E_{\text {ext }}=0 \mathrm{kV} / \mathrm{cm}$, and $I_{1}(z=0)=1 \times 10^{4} \mathrm{~W} / \mathrm{cm}^{2}$. Marked points refer to selected times in Fig. 3.

buildup of the space charge field causes a local decrease in the field, thus resulting in a recovery of the PM condition. When steady state is reached $\left(\tau=\tau_{F}\right)$, the external bias is completely masked by the screening field, while the PV field is not compensated. As a consequence of this partial masking, the system self-stabilizes at a positive mismatched condition (Fig. 5, continuous line arrow).

To explore this regime, we perform a simulation with $T=3^{\circ} \mathrm{C}, \quad E_{\text {ext }}=40 \mathrm{kV} / \mathrm{cm}, \quad E_{\mathrm{PV}}=14 \mathrm{kV} / \mathrm{cm}$, considering again a $\mathrm{FH}$ beam focused to a $15 \mu \mathrm{m}$ diameter at the input face of the $5 \mathrm{~mm}$ long crystal, with an input $\mathrm{FH}$ intensity of $I_{1}(z=0)=1 \times 10^{4} \mathrm{~W} / \mathrm{cm}^{2}$. With these values of temperature and electric field, a moderate negative mismatch exists between $\mathrm{SH}$ and $\mathrm{FH}$ beams as shown in Fig. 5 (continuous line arrow). Figure 6 presents the $\mathrm{SH}$ power evolution at the output face of the sample together with the $\mathrm{SH}$ intensity distribution along propagation at three different times. The simulation clearly shows the influence of self-focusing effect.

At the beginning of the simulation $(\tau=0)$, the beam diffracts and, since PM is not fulfilled, oscillations along the

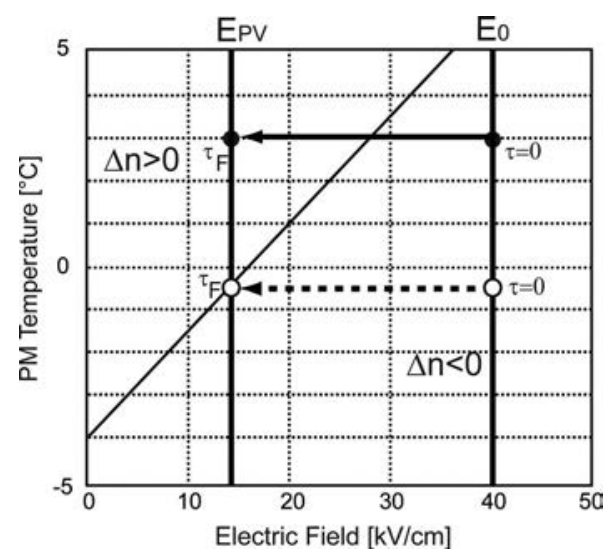

Fig. 5. Variation in the PM condition due to the NLSPM process given by the PR-PV effect. The local masking effect due to PR-PV nonlinearity can induce a switch from a negative to a positive mismatch (continuous line arrow) or lead to perfect matching (dashed line arrow) depending on the initial conditions (temperature and external field).

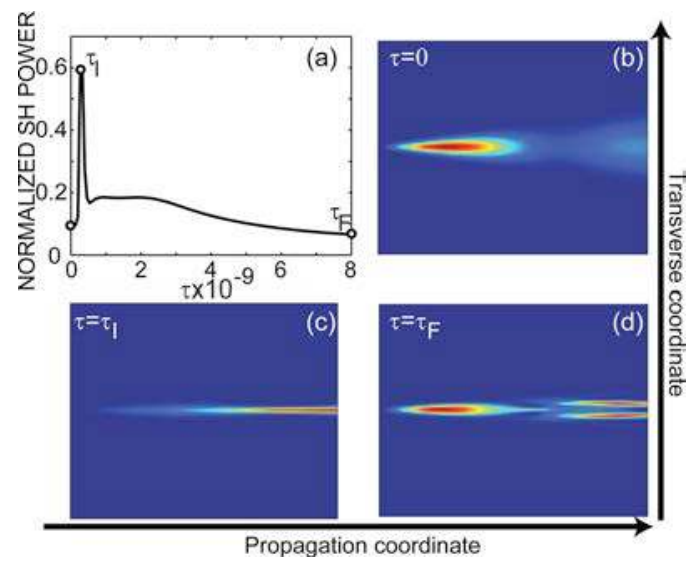

Fig. 6. (Color online) (a) Calculated SH output power evolution as a function of time and (b)-(d) corresponding SH spatial distributions for three different times marked in graph (a). Parameters are $T=3^{\circ} \mathrm{C}, E_{\text {ext }}=40 \mathrm{kV} / \mathrm{cm}, E_{\mathrm{PV}}=14 \mathrm{kV} / \mathrm{cm}, I_{1}(z=0)=1$ $\times 10^{4} \mathrm{~W} / \mathrm{cm}^{2}$

propagation direction can be seen in the $\mathrm{SH}$ intensity distribution plot [Fig. 6(b)]. The buildup of the space charge field then causes a self-focusing effect that efficiently traps the beam; at the same time the initial negative phase mismatch is counterbalanced by the NLSPM process related to the very same space charge field. Figure 6(c) presents the $\mathrm{SH}$ intensity pattern when the perfect PM is attained $\left(\tau=\tau_{I}\right)$. In this case, the $\mathrm{SH}$ intensity monotonically increases along propagation, and the beam is confined in the self-induced waveguide. This situation produces a maximum for the $\mathrm{SH}$ power [Fig. 6(a)], which corresponds to a maximum intensity of $I_{\mathrm{SH}}^{\max }$ $=23.5 \mathrm{~W} / \mathrm{cm}^{2}$ at the output face of the $5 \mathrm{~mm}$ long crystal.

The subsequent evolution of the $\mathrm{SH}$ output power as well as the complex intensity pattern in Fig. 6(d) can be understood considering the propagation of a $\mathrm{FH}$ beam in a (self-induced) waveguide with a time dependent transversal profile [31]. Complex parametric interaction can occur if we consider that several $\mathrm{SH}$ modes can be present in the waveguide. From this perspective, Fig. 6(c) presents the PM occurring between both the $\mathrm{FH}$ and $\mathrm{SH}$ fundamental modes. At $\tau=\tau_{I}$ (perfect PM) the waveguide is perfectly single mode for both frequencies. At $\tau>\tau_{I}$ saturation of the waveguide refractive index profile occurs due to the high intensity ratio between the $\mathrm{SH}$ generated light and the dark irradiance, resulting in a large and deep waveguide profile [10,32]. In this case, several modes can propagate and beat in the induced waveguide, deteriorating the conversion efficiency of the parametric process, as can be seen in Fig. 6(a). A complex intensity pattern appears in the waveguide, testifying to the presence of two $\mathrm{SH}$ even modes beating along propagation as depicted in Fig. 6(d).

However, the detrimental effect of mode beating inside the self-induced waveguide can be avoided if the initial phase mismatch is perfectly compensated by the photoinduced effects. In particular, if the initial temperature $T$ $=-0.5^{\circ} \mathrm{C}$ is chosen (Fig. 5 , dashed line arrow), the applied bias will determine a large negative initial mismatch, which will be perfectly compensated by the local field buildup, driving the system back to perfect PM at steady state. Results from this case are presented in Fig. 7. Due 


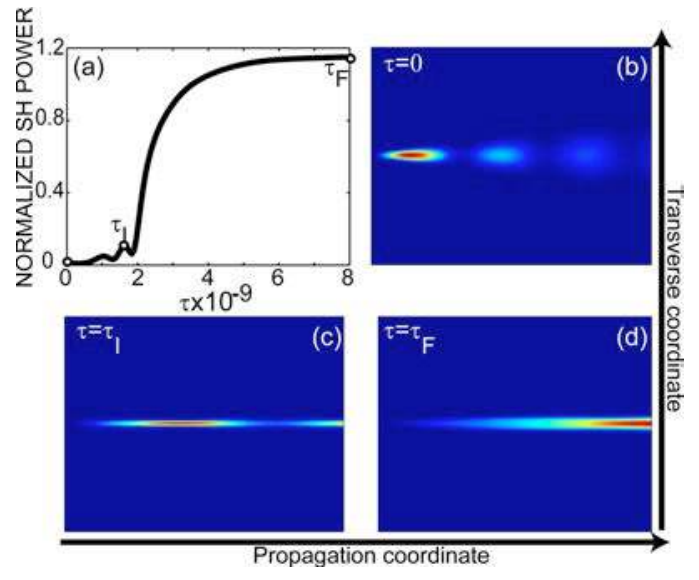

Fig. 7. (Color online) (a) SH output power evolution as a function of time and (b)-(d) corresponding SH spatial distributions for three different times, marked in graph (a). Parameters are $T=-0.5^{\circ} \mathrm{C}, \quad E_{\text {ext }}=40 \mathrm{kV} / \mathrm{cm}, \quad E_{\mathrm{PV}}=14 \mathrm{kV} / \mathrm{cm}, \quad I_{1}(z=0)=1$ $\times 10^{4} \mathrm{~W} / \mathrm{cm}^{2}$.

to the large initial mismatch at $\tau=0$ the $\mathrm{SH}$ intensity distribution presents three periods of oscillation along the propagation axis [Fig. 7(b)]. Progressively, space charge field formation causes beam self-confinement and partially improves the PM condition via the NLSPM process. However, while the tightest confinement is obtained the perfect PM is not yet reached and the optical power is exchanged back and forth between $\mathrm{FH}$ and $\mathrm{SH}$ [Fig. 7(c)], giving an overall low conversion efficiency [Fig. 7(a)]. At steady state $\left(\tau=\tau_{F}\right)$, the optimum PM is achieved between the fundamental $\mathrm{FH}$ and $\mathrm{SH}$ modes, without beating between higher order SH modes despite a broadened multimode waveguide [Fig. 7(d)]. The corresponding SH output power evolution in Fig. 7(a) shows indeed that no oscillation occurs as a consequence of the negligible SH efficiency for higher order modes. In this condition, the calculated $\mathrm{SH}$ maximum intensity reaches $I_{\mathrm{SH}}^{\max }$ $=29.09 \mathrm{~W} / \mathrm{cm}^{2}$ and the SHG optical power generated is higher than the one obtained in the perfect PM regime with absent PR effect. Thus, with this set of parameters, light propagation inside the self-induced PR waveguide improves the SHG performances.

Finally, we show that oscillations observed in Fig. 6(a) can also be suppressed if the waveguide profile is optimized and the PR-PV saturation is avoided. Following the theory on PR waveguides [10,33], one would expect that a single mode waveguide for the SH light could be obtained in a crystal if a large dark irradiance is used. When this dark irradiance is two to three times smaller than the peak intensity of the writing beam, the self-induced waveguide is automatically single mode and a narrow bright PR-PV soliton is obtained at steady state. In typical experiments [2], the dark irradiance is artificially increased using a background illumination, but a temperature raise could also provide an equivalent dark irradiance through thermal generation of charges. In our model we can add in Eq. (2) a spatially uniform incoherent background illumination $I_{b}$ that gives a total dark irradiance $I_{d}=I_{b}+I_{d}^{\prime}$, where $I_{d}^{\prime}$ is now the material dark irradiance. Corresponding numerical results are presented in Fig. 8 with the following parameters: $T=3^{\circ} \mathrm{C}, E_{\text {ext }}=40 \mathrm{kV} / \mathrm{cm}, E_{\mathrm{PV}}$

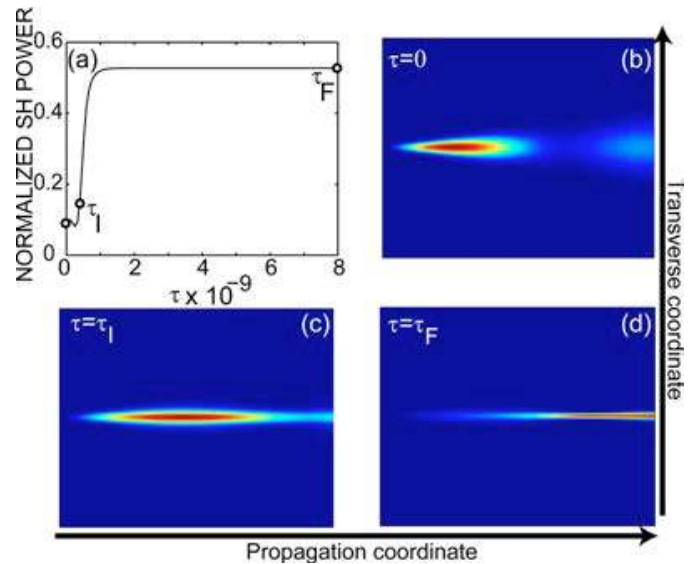

Fig. 8. (Color online) (a) SH output power evolution as a function of time and (b)-(d) corresponding $\mathrm{SH}$ spatial distributions for three different times. Parameters are $T=3^{\circ} \mathrm{C}, E_{\text {ext }}$ $=40 \mathrm{kV} / \mathrm{cm}, E_{\mathrm{PV}}=14 \mathrm{kV} / \mathrm{cm}$. A background illumination is added in order to prevent waveguide saturation $\left(I_{d} \approx I_{b}=5 \mathrm{~W} / \mathrm{cm}^{2}\right)$.

$=14 \mathrm{kV} / \mathrm{cm}, \quad I_{1}(z=0)=1 \times 10^{4} \mathrm{~W} / \mathrm{cm}^{2}, \quad I_{d} \approx I_{b}=5 \mathrm{~W} / \mathrm{cm}^{2}$. Because the parameters are the same as in Fig. 6 except for $I_{d}$, the initial SH intensity distribution over propagation [Fig. 8(b)] is identical as in Fig. 6(b). In Figs. 8(c) and 8(d) a different dynamic can be observed, leading to a selfconfined narrow $\mathrm{SH}$ beam characterized by a tightly focused beam profile [Fig. 8(d)]. The plot of the SH output power time evolution in Fig. 8(a) confirms that a stable propagation is obtained in the waveguide. No oscillation related to multiple higher order $\mathrm{SH}$ modes is observable. The maximum SH intensity obtained with these parameters is $I_{\mathrm{SH}}^{\max }=16.47 \mathrm{~W} / \mathrm{cm}^{2}$. This simulation confirms that the oscillations reported in Fig. 6 are produced by modes beating inside the induced waveguide. We showed that this detrimental effect can be completely removed either by carefully choosing the initial PM condition (case in Fig. 7) or by setting the background irradiance beam to the proper value that leads to the formation of a single mode waveguide (case in Fig. 8). The first approach not only suppresses mode beating inside the self-induced waveguide, but also determines a fair improvement of conversion efficiency.

We would like to emphasize that, by carefully choosing parameters, a spatial soliton could be generated after some formation distance. For instance, if the perfect PM is fulfilled the pump beam can be totally depleted and leave a place to a solitonic $\mathrm{SH}$ beam.

Moreover, it is also valuable to examine the $\mathrm{FH}$ beam behavior along propagation for the configurations leading to stable SH propagation depicted in Figs. 7 and 8. Figure 9(a) presents the initially diffracting $\mathrm{FH}$ beam before the PR effect takes place. When $T=-0.5^{\circ} \mathrm{C}, E_{\text {ext }}=40 \mathrm{kV} / \mathrm{cm}$, $E_{\mathrm{PV}}=14 \mathrm{kV} / \mathrm{cm}$, and negligible $I_{d}$, as for the simulation shown in Fig. 7, we obtain the confined FH beam shown in Fig. 9(b). In this configuration the overlap between the $\mathrm{SH}$ and $\mathrm{FH}$ modes along with the favorable PM conditions leads to the improvement of the frequency doubling efficiency shown in Fig. 7. To the contrary, when a background illumination is used as for the simulation of Fig. 8, a poor overlap is obtained because the $\mathrm{FH}$ beam is weakly guided compared with the $\mathrm{SH}$ beam, leading to a de- 


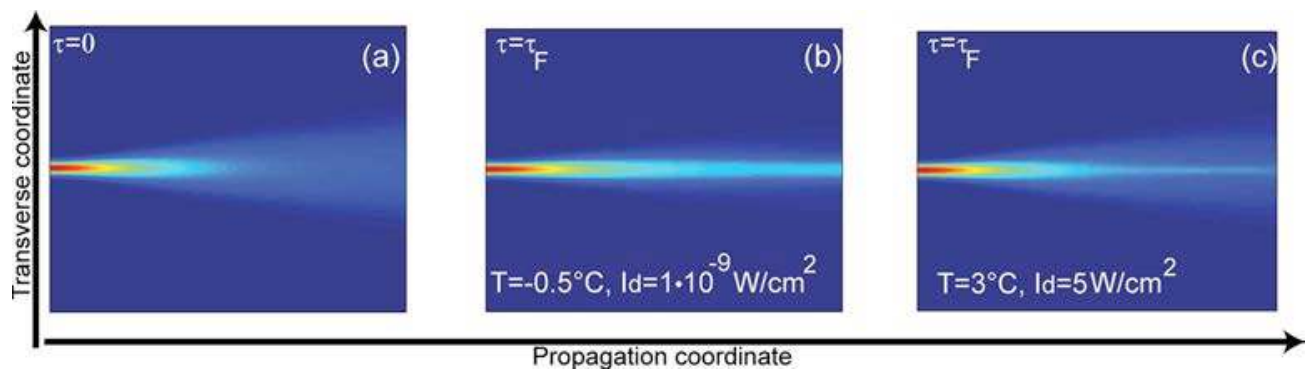

Fig. 9. (Color online) FH spatial distribution (a) at the beginning and at the end of the simulation for (b) $T=-0.5^{\circ} \mathrm{C}, E_{\text {ext }}=40 \mathrm{kV} / \mathrm{cm}$, $E_{\mathrm{PV}}=14 \mathrm{kV} / \mathrm{cm}, I_{1}(z=0)=1 \times 10^{4}, I_{d}=1 \times 10^{-9} \mathrm{~W} / \mathrm{cm}^{2}$ and $(\mathrm{c}) T=3^{\circ} \mathrm{C}, E_{\text {ext }}=40 \mathrm{kV} / \mathrm{cm}, E_{\mathrm{PV}}=14 \mathrm{kV} / \mathrm{cm}, I_{1}(z=0)=1 \times 10^{4}, I_{d}=5 \mathrm{~W} / \mathrm{cm}^{2}$.

creased SH converted output. This result is expected from the PR theory and is related to the stronger confinement of the infrared light in the deep and saturated waveguide profile obtained with negligible dark irradiance [Figs. 7 and $9(\mathrm{~b})$ ] than in the narrower and shallower waveguide formed when the material dark irradiance is artificially increased with an incoherent background illumination [Figs. 8 and $9(\mathrm{c})$ ].

Note that the presented configuration in LN, imposed to obtain the SHG PM, is not favorable to reach an optimum overlap between $\mathrm{SH}$ and $\mathrm{FH}$ guided modes. Indeed the longer $\mathrm{FH}$ wavelength is associated with the lower $r_{13}$ electro-optic coefficient while the shorter wavelength is associated with the stronger $r_{33}$ electro-optic coefficient, which implies that the waveguide seen by the $\mathrm{SH}$ beam is deeper compared with the one seen by the $\mathrm{FH}$ beam. This situation is opposite to an optimum mode overlap.

\section{COMPARISON WITH EXPERIMENTAL RESULTS}

In this section we compare our numerical model with experimental results. Previous investigations reported in [34] demonstrated that 2D self-focusing can be obtained in biased PR-PV LN samples in a noncritical type-I SHG experiment involving a $\mathrm{FH}$ beam ordinary polarized at $1064 \mathrm{~nm}$ and a SH beam extraordinary polarized. Although the experiment involves a $(2+1) \mathrm{D}$ geometry, most observed features are explained by our $(1+1) \mathrm{D}$ model. As in [34], the experiment consists of a $\mathrm{Nd}$ :YAG laser beam focused to an $18 \mu \mathrm{m}$ diameter at the input face of a $2 \mathrm{~cm}$ long photonic grade undoped LN sample electrically biased along the crystal optical $c$ axis. The laser source delivers $1.2 \mathrm{~ns}$ optical pulses at a repetition rate of $12 \mathrm{kHz}$ at $1064 \mathrm{~nm}$. The typical average power launched is $P_{\mathrm{FH}}$ $=760 \mu \mathrm{W}$. Light emerging from the output face of the sample is imaged on a CCD camera in order to monitor the $\mathrm{SH}$ generated beam. In addition the $\mathrm{SH}$ output power evolution is recorded with a power meter. The sample PM temperature is $T_{\mathrm{PM}}=(6 \pm 1)^{\circ} \mathrm{C}$ when an electric field of $E_{\text {ext }}=40 \mathrm{kV} / \mathrm{cm}$ is applied along the $c$ axis. A typical set of parameters used in the experiment is $E_{\text {ext }}=40 \mathrm{kV} / \mathrm{cm}$ and $T=5^{\circ} \mathrm{C}$, giving an initial negative phase mismatch for the $\mathrm{SH}$ process, which can be compensated via the NLSPM effect. Experimental results are depicted in Fig. 10 where the evolutions of the SH output power [Fig. 10(a)] and the SH output mode profile along the $c$ axis at three different times [Figs. 10(b)-10(d)] are reported. The initially diffracting beam in Fig. 10(b) gradually self-traps into a well confined $18 \mu \mathrm{m}$ wide beam [Fig. 10(c)]. At the end of the experiment the SH beam presents a multipeak structure [Fig. 10(d)]. As shown in the power evolution [Fig. 10(a)] an enhancement of the conversion efficiency is quickly obtained due to the combined effect of the beam confinement and the NLSPM effect. This first maximum is reached when a well confined beam is observed [Fig. 10(c)], leading to a $\mathrm{SH}$ generated output of $11 \mu \mathrm{W}$, corresponding to a maximum average intensity of $4.32 \mathrm{~W} / \mathrm{cm}^{2}$. Then the $\mathrm{SH}$ output power oscillates due to the multimode character of the waveguide, as witnessed by the output mode multipeak profile [Fig. 10(d)].

It must be noted that these results obtained in the pulsed regime mode show that self-trapping of the $\mathrm{SH}$ generated beam is possible for $\mathrm{SH}$ peak intensities of the order of $3 \times 10^{5} \mathrm{~W} / \mathrm{cm}^{2}$. For such peak intensities, a pronounced increase in the PV field, has been reported by many authors. This has been explained by suggesting that in a high intensity regime a second impurity center must be considered for the description of the charge transport in LN [35,36]. Recent investigation of the PR effect with high intensity continuous wave (CW) beams has shown the existence of an intensity threshold, above which a dramatic increase in the saturated refractive index takes place [37], determining the onset of optical damage.

The enhancement of the PV field observed in several experiments performed at high intensities would cause a
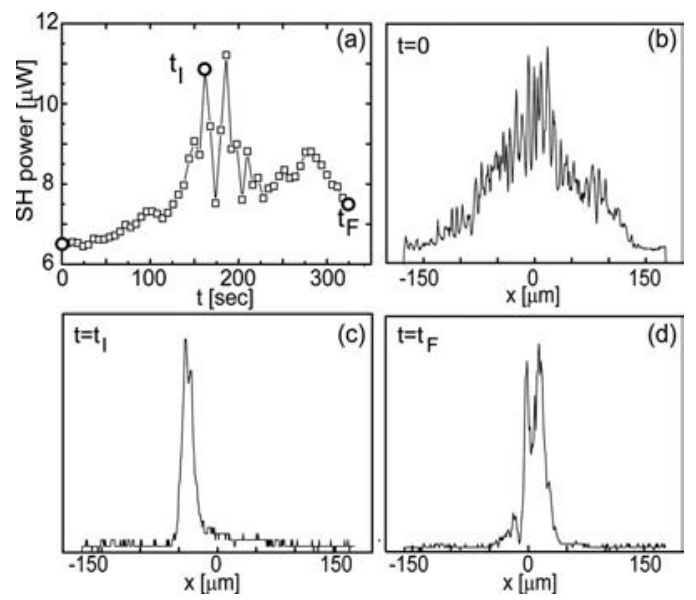

Fig. 10. Experimental results performed with a $40 \mathrm{kV} / \mathrm{cm}$ applied field, a $760 \mu \mathrm{W}$ infrared input power, and a temperature of $5{ }^{\circ} \mathrm{C}$. (a) SH output power evolution and (b)-(d) SH output mode profile along the $c$ axis taken at three different times, as marked in graph (a). 
stronger defocusing effect, which would prevent the observation of self-focusing in $\mathrm{LN}$, because in this case the $\mathrm{PV}$ field can be much larger than the typically employed applied static field. However, in the present experiment the self-focusing leads to a confined beam with a diameter comparable to that obtained using a low intensity $\mathrm{CW}$ visible beam with otherwise similar experimental parameters and with identical values of the external field [18]. It thus demonstrates that, in the conditions of the performed experiment, no intensity dependent increase in the PV field takes place, and that the one center model can be applied for the description of the charge transport in the material.

Because of the low conversion regime obtained experimentally we expect that a PR model valid at low power is sufficient to properly describe the PR response. However for the high power $\mathrm{SH}$ beam it might be necessary to modify the PR model to include, for instance, additional trapping centers. In these conditions it is therefore possible to compare our experimental results obtained in pulsed regime with the theoretical model in the CW keeping in mind that in the present case the PR effect is only dependent on the average intensity of the $\mathrm{SH}$ generated light.

To model the experiment we perform a simulation considering a $2 \mathrm{~cm}$ propagation length and an input diameter of the $\mathrm{FH}$ beam of $18 \mu \mathrm{m}$. We set the PV field to $E_{\mathrm{PV}}$ $=14 \mathrm{kV} / \mathrm{cm}$ and we tune the $\mathrm{FH}$ intensity of the $\mathrm{CW}$ beam in order to generate a $\mathrm{SH}$ maximum intensity comparable to the one obtained in the performed experiment. Moreover we consider an external field of $E_{\text {ext }}=40 \mathrm{kV} / \mathrm{cm}$ with a temperature of $T=5^{\circ} \mathrm{C}$, resulting in an initial negative mismatch as can be seen from Fig. 1 .

The results reported in Fig. 11 present the $\mathrm{SH}$ output power dynamics and the $\mathrm{SH}$ output mode distribution taken at three different characteristic instants. The results are in qualitative agreement with the experiments summarized in Fig. 10 for both the SH power and the mode profile evolutions. At first, the initially diffracting

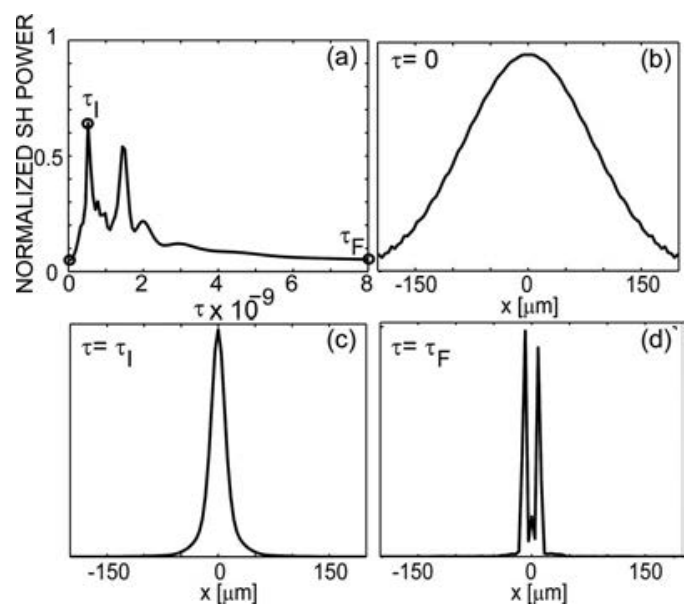

Fig. 11. Results from a simulation performed with a $40 \mathrm{kV} / \mathrm{cm}$ external field, at a temperature of $5^{\circ} \mathrm{C}$, with a propagation of 2 $\mathrm{cm}$ and an input $\mathrm{FH}$ beam diameter of $18 \mu \mathrm{m}$. The PV field is $E_{\mathrm{PV}}=14 \mathrm{kV} / \mathrm{cm}$ and $I_{1}(z=0)=2100 \mathrm{~W} / \mathrm{cm}^{2}$. In (a) the SH output power evolution as a function of time is represented. In (b)-(d) the $\mathrm{SH}$ output mode profile along the $c$-axis direction taken at three different times, as reported in graph (a), is presented. beam [Fig. 11(b)] is associated with a low conversion efficiency [Fig. 11(a)]. The PR effect induces subsequently a self-trapping that leads to a single mode confined $14 \mu \mathrm{m}$ wide beam as seen in Fig. 11(c). When this strong confinement is reached the $\mathrm{SH}$ power attains a maximum value [see Fig. 11(a)]. The conversion efficiency then rapidly decreases, which indicates a degradation of the PM conditions for the fundamental mode of the structure [Fig. 11(a)]. As the PR effect continues to progress, $\mathrm{SH}$ power oscillations appear along with a complicated mode structure that ends up with the multihump structure shown in Fig. 11(d). The overall behavior is thus similar to the experimental results. Note also that the dielectric relaxation time calculated for the present simulation gives $T_{D} \approx 1 \times 10^{11} \mathrm{~s}$; therefore the temporal scale for the plot shown in Fig. 11 is of the same order of magnitude as the dynamics observed experimentally. The multimode character of the waveguide is confirmed by the intensity profile shown in Fig. 11(d). Therefore, the numerical simulation confirms that $\mathrm{SH}$ power oscillations observed experimentally are related to modes beating inside the self-induced waveguide. Moreover this study reveals that the observed dynamics are dictated by the waveguide profile evolution and by the NLSPM process.

\section{CONCLUSIONS}

In this paper we studied the temporal dynamics of second harmonic generation (SHG) in a photorefractive photovoltaic (PR-PV) crystal by solving numerically the Schrödinger coupled equations in conjunction with the time dependent photorefractive (PR) equation expressing the nonlinear refractive index change. We first considered the case of the SHG in lithium niobate (LN) crystals without an external applied field, in which the defocusing PR effect due to the photovoltaic (PV) current causes a local modification of the phase matching (PM) condition. As a consequence the nonlinear self-phase-matching (NLSPM) is responsible for the variation in the green output power during the exposure time. The results agree with a previously reported experimental analysis [17].

Our simulations also confirm that the application of an external field along the crystal $c$ axis allows a selftrapping for both the fundamental and second harmonic (SH) beams. This configuration makes beams selfconfined in a single mode waveguide provided that parameters such as temperature and intensity to dark irradiance are properly chosen. Moreover, a fair improvement of the SH generation is realized when the fundamental beam is guided in the deep and saturated waveguide and at the same time a perfect PM is achieved between the fundamental infrared and green modes. Otherwise our analysis demonstrates that an oscillating behavior is obtained for the $\mathrm{SH}$ output power evolution because the selfinduced waveguide allows propagation of several $\mathrm{SH}$ guided modes, which induce mode beating.

Finally, the model developed here is compared with experimental results on $2 \mathrm{D}$ self-focusing through the SHG in LN. The experimentally observed dynamics and the converted output power evolution can be explained in terms of mode beating inside the self-induced waveguide. The presented model offers the possibility to determine 
experimental conditions that are able to reach efficient and stable SHGs by taking advantage of the PR effect. A more refined description would necessitate the development of a full 3D model that takes into account the two transverse dimensions of the beam and possibly the presence of an additional trap level.

\section{ACKNOWLEDGMENTS}

Financial support from Universitá Italo-Francese (UIF-UFI) through VINCI 2005 program and from Italian Project PRIN 2005 "Amplificatori e sorgenti laser integrati in guide solitoniche in $\mathrm{Er}: \mathrm{LiNbO}_{3}$ " is acknowledged.

\section{REFERENCES}

1. P. Yeh, Introduction to Photorefractive Nonlinear Optics (Wiley-Interscience, 1993).

2. M.-F. Shih, M. Segev, G. C. Valley, G. Salamo, B. Crosignani, and P. Di Porto, "Observation of twodimensional steady-state photorefractive screening solitons," Electron. Lett. 31, 826-827 (1995).

3. M. Mitchell, M. Segev, and D. C. Christodoulides, "Observation of multihump multimode solitons," Phys. Rev. Lett. 80, 4657-4660 (1998).

4. D. Neshev, E. Ostrovskaya, Y. Kivshar, and W. Krolikowski, "Spatial solitons in optically induced gratings," Opt. Lett. 28, 710-712 (2003).

5. J. W. Fleischer, T. Carmon, M. Segev, N. K. Efremidis, and D. N. Christodoulides, "Observation of discrete solitons in optically induced real time waveguide arrays," Phys. Rev. Lett. 90, 023902 (2003).

6. Z. Chen and K. McCarthy, "Spatial soliton pixels from partially incoherent light," Opt. Lett. 27, 2019-2021 (2002).

7. D. Trager, N. Sagemerten, and C. Denz, "Guiding of dynamically modulated signals in arrays of photorefractive spatial solitons," IEEE J. Sel. Top. Quantum Electron. 12, 383-387 (2006)

8. V. Coda, M. Chauvet, F. Pettazzi, and E. Fazio, "3-D integrated optical interconnect induced by self-focused beam," Electron. Lett. 42, 463-465 (2006).

9. M. Klotz, H. Meng, G. J. Salamo, M. Segev, and S. R. Montgomery, "Fixing the photorefractive soliton," Opt. Lett. 24, 77-79 (1999).

10. M.-F. Shih, Z. Chen, M. Mitchell, M. Segev, H. Lee, R. S. Feigelson, and J. P. Wilde, "Waveguides induced by photorefractive screening solitons," J. Opt. Soc. Am. B 14, 3091-3101 (1997).

11. W. Sohler and H. Suche, "Second-harmonic generation in Ti-diffused $\mathrm{LiNbO}_{3}$ optical waveguides with $25 \%$ conversion efficiency," Appl. Phys. Lett. 33, 518-519 (1978).

12. M. H. Chou, J. Hauden, M. A. Arbore, and M. M. Fejer, "1.5- $\mu \mathrm{m}$-band wavelength conversion based on differencefrequency generation in $\mathrm{LiNbO}_{3}$ waveguides with integrated coupling structures," Opt. Lett. 23, 1004-1006 (1998).

13. R. Regener and W. Sohler, "Efficient second-harmonic generation in $\mathrm{Ti}: \mathrm{LiNbO}_{3}$ channel waveguide resonators," $\mathrm{J}$. Opt. Soc. Am. B 5, 267-277 (1988).

14. R. Schiek, Y. Baek, and G. I. Stegeman, "Second-harmonic generation and cascaded nonlinearity in titaniumindiffused lithium niobate channel waveguides," J. Opt. Soc. Am. B 15, 2255-2568 (1998).

15. G. Assanto and G. I. Stegeman, "Simple physics of quadratic spatial solitons," Opt. Express 10, 388-396 (2002).

16. A. V. Buryak and Y. S. Kivshar, "Spatial optical solitons governed by quadratic nonlinearity," Opt. Lett. 19, 1612-1614 (1994).

17. S. Orlov, A. Yariv, and M. Segev, "Nonlinear self-phase matching of optical second harmonic generation in lithium niobate," Appl. Phys. Lett. 68, 1610-1612 (1996).

18. E. Fazio, F. Renzi, R. Rinaldi, M. Bertolotti, M. Chauvet, W. Ramadan, A. Petris, and V. I. Vlad, "Screening-photovoltaic bright solitons in lithium niobate and associated singlemode waveguides," Appl. Phys. Lett. 85, 2193-2195 (2004).

19. C. Lou, J. Xu, H. Qiao, X. Zhang, Y. Chen, and Z. Chen, "Enhanced second-harmonic generation by means of high power confinement in a photovoltaic soliton-induced waveguide," Opt. Lett. 29, 953-955 (2004).

20. J. Liu and L. Keqing, "Screening-photovoltaic spatial solitons in biased photovoltaic-photorefractive crystals and their self-deflection,” J. Opt. Soc. Am. B 16, 550-555 (1999).

21. A. A. Zozulya and D. Z. Anderson, "Propagation of an optical beam in a photorefractive medium in the presence of a photogalvanic nonlinearity or an externally applied electric field," Phys. Rev. A 51, 1520-1531 (1995).

22. S. Lan, M.-F. Shih, G. Mizell, J. A. Giordmaine, Z. Chen, C. Anastassiou, J. Martin, and M. Segev, "Second-harmonic generation in waveguides induced by photorefractive spatial solitons," Opt. Lett. 24, 1145-1147 (1999).

23. A. D. Boardman, W. Ileki, and Y. Liu, "Spatial solitons in a photorefractive medium sustaining second-harmonic generation,” J. Opt. Soc. Am. B 19, 832-838 (2002).

24. R. L. Byer, Y. K. Park, R. S. Feigelson, and W. L. Kway, "Efficient second-harmonic generation of Nd:YAG laser radiation using warm phasematching $\mathrm{LiNbO}_{3}$," Appl. Phys. Lett. 39, 17-19 (1981).

25. N. Uesugi, K. Daikoku, and K. Kubota, "Electric field tuning of second-harmonic generation in a threedimensional $\mathrm{LiNbO}_{3}$ optical waveguide," Appl. Phys. Lett. 34, 60-62 (1979).

26. U. Schlarb and K. Betzler, "Refractive indices of lithium niobate as a function of temperature, wavelength, and composition: a generalized fit," Phys. Rev. B 48, 15613-15620 (1993).

27. D. N. Christodoulides and M. I. Carvalho, "Bright, dark, and gray spatial soliton states in photorefractive media," J. Opt. Soc. Am. B 12, 1628-1633 (1995).

28. M. Chauvet, V. Coda, H. Maillotte, E. Fazio, and G. Salamo, "Large self-deflection of soliton beams in $\mathrm{LiNbO}_{3}$," Opt. Lett. 30, 1977-1979 (2005).

29. R. C. Miller and A. Savage, "Temperature dependence of the optical properties of ferroelectric $\mathrm{LiNbO}_{3}$ and $\mathrm{LiTaO}_{3}$," Appl. Phys. Lett. 9, 169-171 (1966).

30. T. Fujihara, M. Tokuue, S. Umegaki, T. Sassa, and M. Yokoyama, "Formation of an anti-guide structure and observation of enhanced SHG in photorefractive materials," Opt. Mater. 21, 51-54 (2003).

31. A. W. Snyder, S. J. Hewlett, and D. J. Mitchell, "Periodic solitons in optics," Phys. Rev. E 51, 6297-6300 (1995).

32. N. Fressengeas, M. Maufoy, and K. Kugel, "Temporal behavior of bidimensional photorefractive bright spatial solitons," Phys. Rev. E 54, 6866-6875 (1996).

33. K. Lu, M.-Z. Zhang, W. Zhao, Y.-L. Yang, Y.-H. Zhang, X.-M. Liu, Y.-P. Zhang, and J.-P. Song, "Waveguides induced by screening-photovoltaic solitons in biased photorefractive-photovoltaic crystals," Chin. Phys. Lett. 23, 2770-2772 (2006).

34. F. Pettazzi, V. Coda, M. Chauvet, and E. Fazio, "Frequencydoubling in self-induced waveguides in lithium niobate," Opt. Commun. 272, 238-241 (2007).

35. F. Jermann, M. Simon, and E. Kratzig, "Photorefractive properties of congruent and stoichiometric lithium niobate at high light intensities," J. Opt. Soc. Am. B 12, 2066-2070 (1995).

36. K. Buse, "Light-induced charge transport processes in photorefractive crystals II: materials," Appl. Phys. B 64, 391-407 (1997).

37. M. Carrascosa, J. Villaroel, J. Carnicero, A. GarciaCabanes, and J. M. Cabrera, "Understanding light intensity thresholds for catastrophic optical damage in $\mathrm{LiNbO}_{3}$," Opt. Express 16, 115-120 (2008). 